\title{
LAMOST 2D pipeline
}

\author{
BAI Zhongrui \\ National Astronomic Observatories, Chinese Academy of Sciences, \\ A20 Road Datun, Chaoyang Distinct, Beijing, China \\ email: zrbai@bao.ac.cn
}

Abstract. This paper describes the 2-dimensional data reduction of the LAMOST pilot survey.

Keywords. LAMOST, 2D pipeline, data reduction

The Large Sky Area Multi-Object Fiber Spectroscopic Telescope (LAMOST, also called the Guoshoujing Telescope) has the capability of taking 4000 spectra with resolution $(\mathrm{R}=1800)$ simultaneously in a single exposure (Cui et al. 2012). The LAMOST 2D pipeline aims to extract spectra from CCD images, and to calibrate them, including wavelength calibration, sky subtraction, flux calibration and co-addition. A number of procedures from the spectro2d pipeline of SDSS are used (Stoughton et al. 2012).

The pipeline has been run successfully on all spectroscopic data obtained during LAMOST Pilot Surveys, from October 24, 2010 to June 17, 2012(Luo et al. 2012).

Flux extraction. The raw data is obtained by 32 EEV CCD chips (Wei \& Stover 1996)(Fig. 1). The fiber position on the CCD is traced in flat-field spectra. The centroid of one fiber in row direction is fitted by a polynomial along column direction. The profile of one fiber in row direction is assumed to be a Gaussian-like profile, which is characterized by $y=a e^{-(x-b)^{d} / d c^{d}}$. The coefficients are calculated by flat-field spectra as used for other exposures.

Wavelength calibration. The arc lamp spectra are extracted, and the centroids of the arc emission lines are measured, to which we fit a Legendre polynomial as a function between wavelengths and pixels. The wavelength error of both sides is less than $0.1 \AA$.

Sky subtraction. More than 20 fibers are used to model the background of a specific region of the sky. A supersky model is constructed by performing iterative fits to the extracted spectra associated with blank sky positions. For each fiber in that region, the supersky background is subtracted. Telluric absorption in four bands is removed.

Flux calibration. About 5 of the fibers are selected to point to flux standard stars. The sky-subtracted flux is divided by the best-fit model at the best-fit redshift. These ratios are called the calibration vectors. The set of flux-calibration vectors for an entire spectrograph are combined using B-splines to compute an average flux-calibration vector per CCD. Finally, for each object in the individual exposures, the spectra from the red and blue sides are combined. The combined spectra are resampled in constant-velocity pixels, with a pixel scale of $69 \mathrm{~km} \mathrm{~s}^{-1}$.

Conclusion. This pipeline has been used for the LAMOST Pilot Survey in which we released about 316,000 spectra. The wavelength calibration is accurate to $10 \mathrm{~km} \mathrm{~s}^{-1}$ or better. The sky residual is less than $10 \%$. 


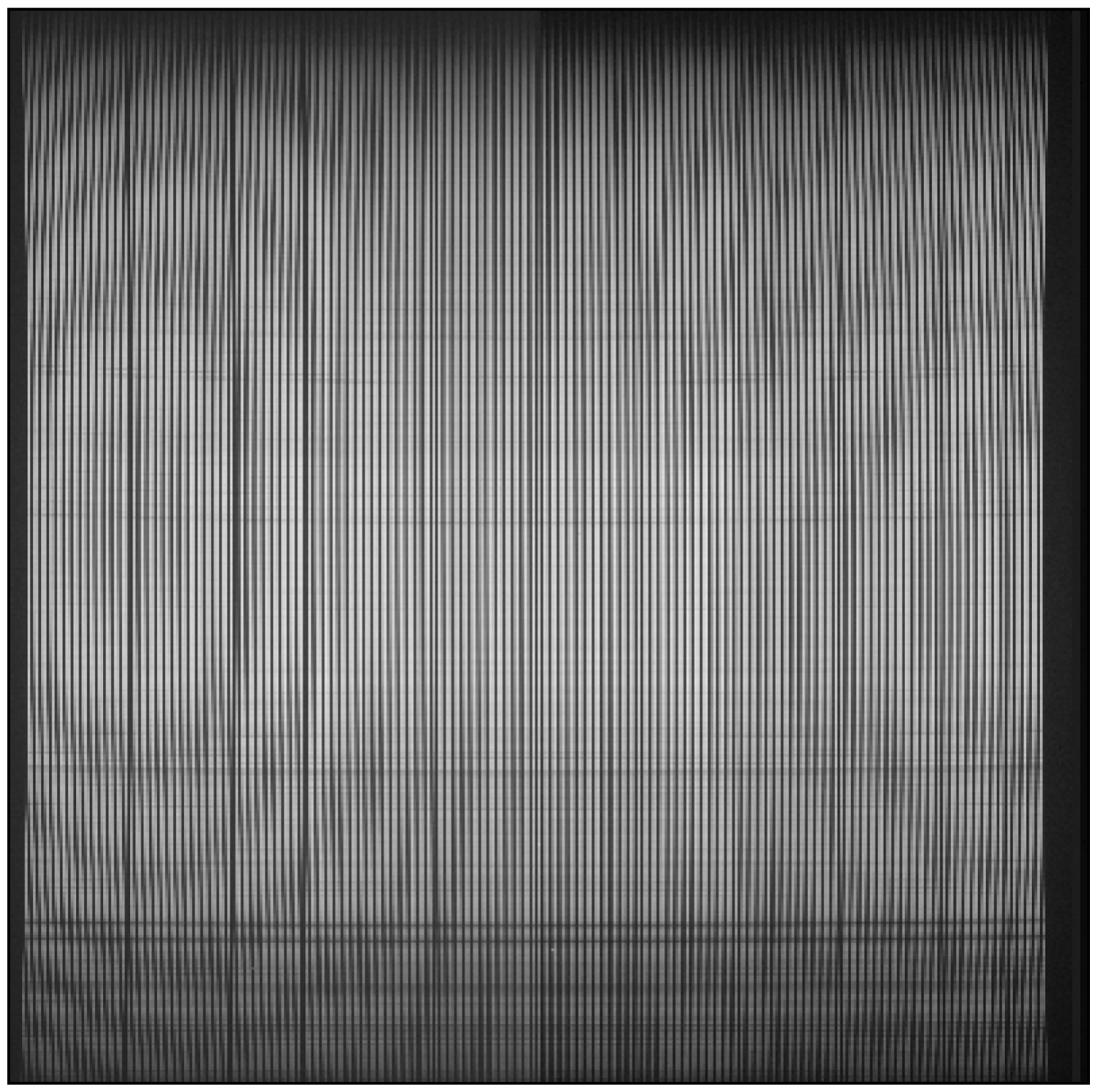

Figure 1. Raw data on the CCD.

\section{References}

Cui, X., Zhao, Y., Chu, Y., et al. 2012, RAA, 12, 1197

Stoughton, C., Lupton, R. H., et al. 2012, AJ, 123, 485

Luo, A., Zhang, H., Zhao, Y., et al. 2012, RAA, 12, 1243

Wei, M. \& Stover R. J. 1996, Solid State Sensor Arrays and CCD Cameras(San Jose), 226 